\title{
Limiting Physical-Layer Attack Propagation with Power Equalization Placement in Transparent WDM Networks
}

\author{
Amornrat Jirattigalachote ${ }^{1}$, Nina Skorin-Kapov ${ }^{2}$, Marija Furdek ${ }^{2}$, Jiajia Chen ${ }^{1}$, Paolo Monti ${ }^{1}$, \\ and Lena Wosinska ${ }^{1}$ \\ ${ }^{1}$ Royal Institute of Technology KTH/ICT, Isafjordsgatan 22, Electrum 229, 16440 Kista, Sweden \\ ${ }^{2}$ Department of Telecommunications, FER, University of Zagreb, Unska 3, 10000 Zagreb, Croatia \\ \{amornrat, jiajiac,pmonti,wosinska\}@kth.se; \{nina.skorin-kapov,marija.furdek\}@fer.hr \\ $\mathbf{1}^{\text {st }}$ author is a student
}

\begin{abstract}
We propose and evaluate a Greedy Randomized Adaptive Search Procedure applied to minimize the number of power equalizers (GRASP-PE) needed to limit the propagation of high-power jamming attacks in WDM networks. Simulation results show that GRASP-PE significantly reduces the required number of power equalizers when compared to a greedy approach.
\end{abstract}

\section{Introduction}

In transparent optical networks (TONs) based on Wavelength Division Multiplexing (WDM) technology, the signal is transported from source to destination entirely in the optical domain through all-optical channels called lightpaths. The absence of optoelectronic processing, i.e., $\mathrm{O} / \mathrm{E} / \mathrm{O}$ conversion, of a signal at intermediate nodes along a lightpath enables transparency to bit rate, signal format, and protocols. However, transparency makes TONs highly vulnerable to physical-layer attacks. An attack-aware routing algorithm based on tabu search has been proposed in [1] to minimize the possible disruption caused by a high-power jamming attack by minimizing the maximum number of lightpaths that share a common fiber link in the network. In [1] it was assumed that attacks, once injected, can propagate unhindered in the network.

On the other hand, the propagation of high-power jamming attacks causing gain competition in optical amplifiers and inter-channel crosstalk in fiber links can be efficiently prevented by installation of power equalizers in the network nodes. However, due to the high cost of such equipment, the number of power equalizers should be minimized and their placement in the network should be done in such a way that the attack propagation for a given routing scheme, especially those that are not attack-aware, is minimized.

This paper proposes $(i)$ a node architecture with power equalizers (based on wavelength-selective attenuators [2]), and (ii) a Greedy Randomized Adaptive Search Procedure (GRASP) [3] to solve the power equalization placement problem so that the propagation of high-power jamming attacks is reduced by using a minimum number of power equalizers.

\section{Performance Evaluation}

This section first describes the proposed Greedy Randomized Adaptive Search Procedure for the power equalization placement problem (GRASP-PE), and then presents the experimental setup and the simulation results. It is assumed that an attack can be inserted as a high-power jamming signal anywhere along a legitimate lightpath. A jamming attack is able to propagate to all lightpaths that share a physical link with the attacked lightpath until a power equalizer is reached. A lightpath can be then divided into sub-lightpaths if it passes through the intermediate node equipped with a power equalizer, i.e., a lightpath traversing $n$ intermediate nodes equipped with power equalizers is divided into $n+1$ sub-lightpaths. The maximum Lightpath Attack Radius ( $\max L A R$ ) provides a measure of how much a jamming attack can spread. max $L A R$ is defined as the maximum number of (sub) lightpaths that any one (sub) lightpath shares a common fiber link with. Fig. 1 shows the proposed node architecture to obtain power equalization via wavelength-selective attenuators.

Given a network topology and a routing scheme, the objective of the GRASP-PE algorithm is to place a minimum number of power equalizers in such a way as to reduce the $\max L A R$ value to a desired level. GRASPPE consists of two main steps; (1) a construction phase, where a feasible solution to the problem is built, and (2) a local search phase, where neighboring solutions are iteratively searched until the first local optimum is reached in order to reduce the number of power equalizers needed without increasing the maxLAR. The pseudo code of GRASP-PE is shown in Fig. 2. For benchmarking purpose, a greedy approach was also considered where the power equalizers are placed at each node, starting from the most loaded, until the maxLAR values reach the desired level.

The 30-node reference European COST 266 network [4] is used to evaluate the performance of GRASP-PE through simulations. Three routing schemes are considered, (1) shortest path routing, (2) a tabu-search heuristic routing algorithm aimed at minimizing $\max L A R$ [1], and (3) $k$-shortest path routing [5]. The stopping condition for GRASP-PE is maxLAR = network congestion (i.e., the maximum number of lightpaths routed over any one physical link in the network), for each of the specific routing schemes, which represents the lower bound for 
$\max L A R$. GRASP-PE was run for 12 different virtual topologies, i.e. sets of lightpaths, with the following parameters: GraspIteration $=100, R C L \_$size $=10$.

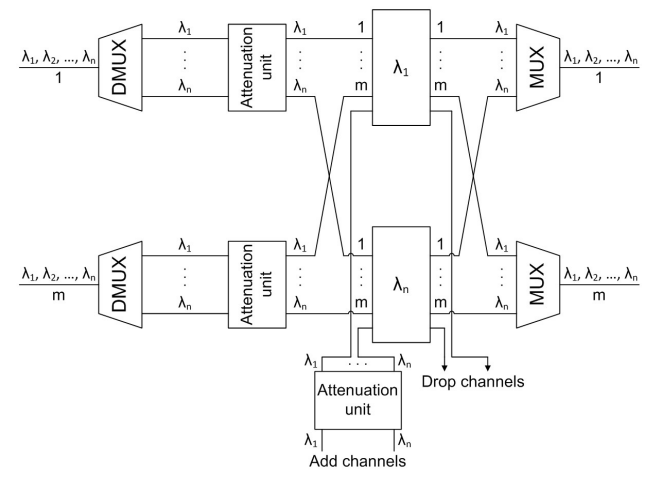

Fig. 1. Architecture of a node with power equalization.

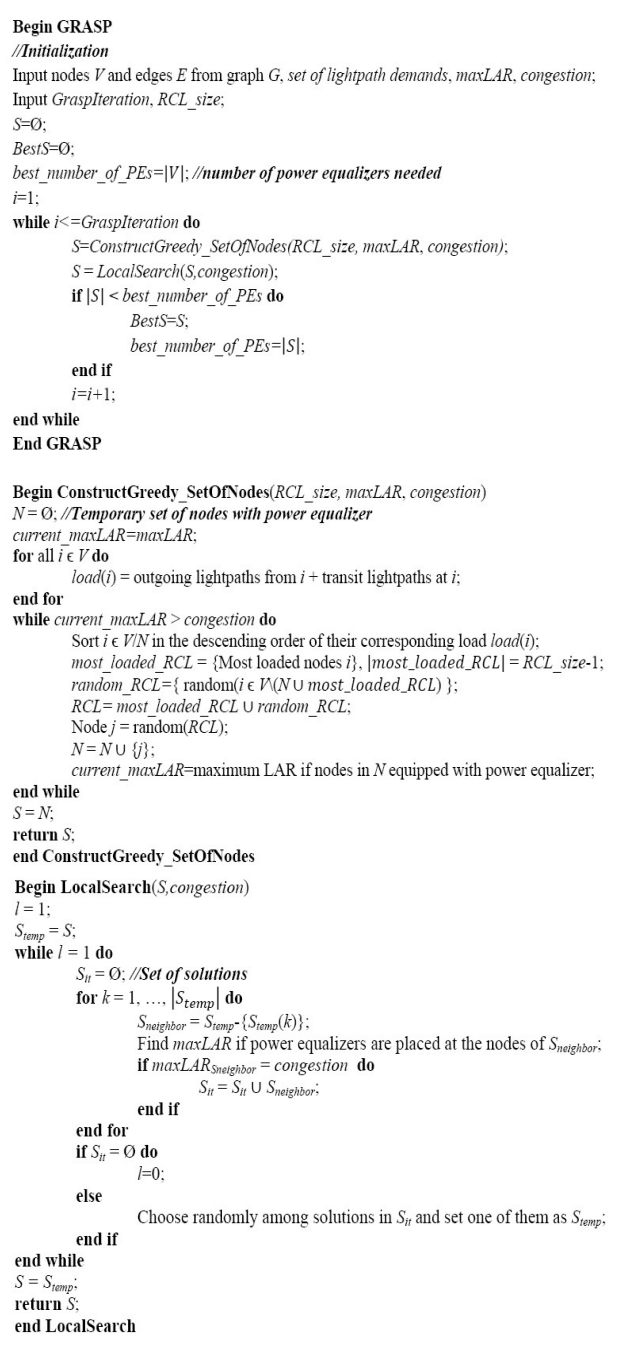

(d) $\max L A R$ vs. Congestion

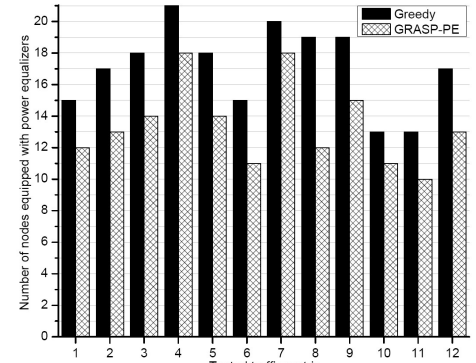

(b) Tabu search

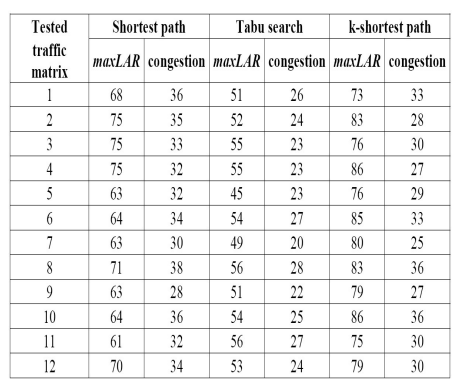

(c) k-shortest path

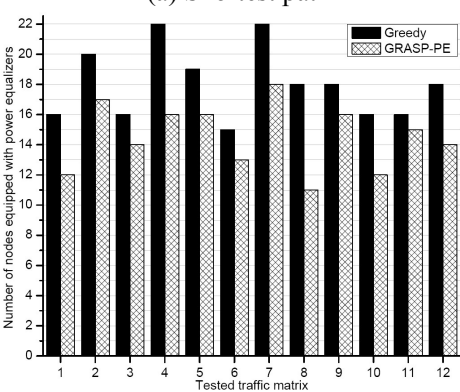

Fig. 3. Number of nodes equipped with power equalizers and maxLAR vs. congestion. Fig. 2. Pseudocode of the GRASP-PE algorithm.

Fig. 3 (a-c) shows the number of nodes equipped with power equalizers that are necessary to reduce the $\max L A R$ to the value of the network congestion. Compared to the greedy approach, GRASP-PE significantly decreases the number of power equalizers needed in all tested virtual topologies. This reduction is most essential in the tabu-search routing algorithm, which aims to minimize the maxLAR and provides lower congestion than the other routing schemes (see Fig. 3 (d)).

\section{Conclusions}

This paper proposes and evaluates the performance of a Greedy Randomized Adaptive Search Procedure applied to the power equalization placement problem (GRASP-PE). The objective of GRASP-PE is to limit the propagation of high-power jamming attacks using a minimum number of power equalizers. A node architecture integrated with wavelength-selective attenuators working as power equalizers is also proposed. The simulation results show that GRASP-PE, compared to the greedy approach, requires a significantly smaller number of power equalizers to equally reduce the maximum potential damage of jamming attacks on the lightpaths.

\section{References}

[1] N. Skorin-Kapov et al., IEEE/ACM Transactions on Networking, vol. 18, no. 3, pp. 750-760, June 2010.

[2] A. Mocki et al., in Proc. IEEE International Telecommunications Symposium, Sep. 2006.

[3] M.G.C. Resende et al., Handbook of Metaheuristics, pp. 219-249, Springer, New York, 2003.

[4] R. Inkret et al., Extended final report of COST Action 266, Faculty of Elect. Eng. Comput., Univ. Zagreb, pp. 19-21, 2003.

[5] E.Q.V. Martins et al., 4OR: A Quarterly Journal of Operations Research, vol. 1, no. 2, pp. 121-133, June 2003. 